\title{
Adenoviral-Mediated Modulation of Sim1 Expression in the Paraventricular Nucleus Affects Food Intake
}

\author{
Chun Yang, ${ }^{1}$ David Gagnon, ${ }^{2,3}$ Pascal Vachon, ${ }^{4}$ André Tremblay, ${ }^{1}$ Emile Levy, ${ }^{1}$ Bernard Massie,,${ }^{2,3}$ and \\ Jacques L. Michaud ${ }^{1}$ \\ ${ }^{1}$ Centre de Recherche, Hôpital Sainte-Justine, Montréal, Québec, Canada H3T 1C5, ${ }^{2}$ Institut de Recherche en Biotechnologie, Conseil National de Recherche \\ du Canada, Montréal, Québec, Canada H4P 2R2, ${ }^{3}$ Département de Microbiologie et Immunologie, Faculté de Médecine, Université de Montréal, Montréal, \\ Québec, Canada H3C 3T4, and 4Faculté de Médecine Vétérinaire, Université de Montréal, Saint-Hyacinthe, Québec, Canada J2S 7C6
}

Haploinsufficency of Sim1, which codes for a basic helix-loop-helix-PAS (PER-ARNT-SIM) transcription factor, causes hyperphagia in mice and humans, without decrease in energy expenditure. Sim1 is expressed in several areas of the brain, including the developing and postnatal paraventricular nucleus (PVN), a region of the hypothalamus that controls food intake. We have previously found that the number of PVN cells is decreased in Sim $1^{+/-}$mice, suggesting that their hyperphagia is caused by a developmental mechanism. However, the possibility that Siml functions in the postnatal PVN to control food intake cannot be ruled out. To explore this hypothesis, we used adenoviral vectors to modulate Sim1 expression in the postnatal PVN of wild-type mice. Unilateral stereotaxic injection into the PVN of an adenoviral vector producing a short hairpin RNA directed against Sim1 resulted in a significant increase in food intake, which peaked to $22 \% 6 \mathrm{~d}$ after the procedure, compared with the injection of a control virus. In contrast, injection of an adenovirus that expresses Sim 1 induced a decrease in food intake that was maximal on the seventh day after the procedure, reaching $20 \%$. The impact of bilateral injections of these vectors into the PVN was not greater than that of unilateral injections. Together, these results strongly suggest that Sim1 functions along a physiological pathway to control food intake.

Key words: hypothalamus; mice; energy; transcription factor; development; obesity

\section{Introduction}

The basic helix-loop-helix-PAS (PER-ARNT-SIM) transcription factor SIM1 is required for the development of the paraventricular nucleus (PVN) of the hypothalamus (Michaud et al., 1998). In the absence of Sim1, virtually all cells of the PVN are missing and mice die shortly after birth, presumably because of these defects. In contrast, mice with only one copy of a $\operatorname{Sim} 1$ mutant allele survive but show increased weight gain after 4 weeks of age (Michaud et al., 2001; Holder et al., 2004). Previous studies suggest that Sim 1 haploinsufficiency induces obesity by increasing food intake, without decreasing energy expenditure (Michaud et al., 2001; Holder et al., 2004) (Duplan et al., unpublished observations). A decrease in SIM1 also causes obesity in humans, presumably by inducing hyperphagia (Holder et al., 2000).

The PVN is a critical regulator of energy balance, controlling both feeding and energy expenditure. It does so by acting as a component of a circuit that links the arcuate nucleus, in which leptin functions, and preganglionic neurons of the autonomic nervous system located in the brainstem and in the spinal cord (Swanson and Sawchenko, 1983). Thus, it is plausible that Sim1

Received Feb. 15, 2006; revised May 22, 2006; accepted May 23, 2006.

This work was supported by Canadian Institutes of Health Research Grant MOP-15458. J.L.M. is a Scientist of the Fonds de la Recherche en Santé du Québec.

Correspondence should be addressed to Dr. Jacques L. Michaud, Research Center, Hôpital Sainte-Justine, 3175 Côte Sainte-Catherine, Montréal, Québec, Canada H3T 1C5. E-mail: jacques.michaud@recherche-ste-justine.qc.ca. DOI:10.1523/JNEUROSCI.0672-06.2006

Copyright $\odot 2006$ Society for Neuroscience $\quad$ 0270-6474/06/267116-05\$15.00/0 haploinsufficiency causes hyperphagia by interfering with the function of the PVN. Indeed, we found that the number of PVN cells is decreased by $24 \%$ in Sim 1 heterozygous mice, raising the possibility that their hyperphagia has a developmental origin (Michaud et al., 2001). However, because Sim1 is expressed continuously in the postnatal PVN, it could also function physiologically to control food intake (Michaud et al., 2001). Alternatively, Sim 1 could control food intake by acting in other regions of the developing and adult brain in which it is expressed, such as the nucleus of the lateral olfactory tract of the amygdala and the ventrolateral hypothalamus (Balthasar et al., 2005). To determine by which of these mechanisms Sim1 haploinsufficiency increases food intake, we used adenoviral vectors to alter Sim 1 expression levels in the PVN of wild-type mice.

\section{Materials and Methods}

Production of adenoviruses. Three defective serotype 5 adenoviruses (AdshRNA-Sim1, Ad-Sim1, and Ad-CTRL) were generated through homologous recombination between protease S gene (PS)-expressing shuttle vectors and PS-deleted Ad5 genome cleaved in E1 (Elahi et al., 2002; Ogorelkova et al., 2004). Construction of shuttle vectors was performed as follows:

(1) For production of the Ad-shRNA-Sim1 virus, we first identified, using the Oligoengine (Seattle, WA) software, two sequences of $19 \mathrm{nu}-$ cleotides within the Sim 1 coding region of the mouse to design templates for the production of short hairpin RNAs (shRNAs). Briefly, an oligonucleotide containing either one of these 19-nucleotide sequences separated by a short spacer from the reverse complement of the same se- 
quence and a five thymidine termination signal was cloned in the pSUPER expression vector system downstream of an H1 RNA polymerase III promoter (Oligoengine). The efficacy of these constructs in reducing the Sim1 transcript was tested in cultured cell systems. The region containing the $\mathrm{H} 1$ promoter and the shRNA template that was the most efficient ( $5^{\prime}$ - CTCGGCTCTCATCTACTCC-3') was then amplified by PCR from the pSUPER vector and cloned into the shuttle vector pAdCMV5/PS (D. Gagnon and B. Massie, unpublished observations). This vector also contains, downstream of the shRNA template, a red fluorescent protein (RFP)-producing cassette under the control of a human cytomegalovirus (CMV)-immediate-early promoter.

(2) For production of the Ad-Sim1 virus, a mouse Sim1 cDNA, which was modified to contain a Kozak translation initiation sequence, was cloned into the pAdCMV5-IRES-GFP/PS vector that coexpresses Sim1 and Gfp from the human CMV-immediate-early promoter using an internal ribosome entry site (D. Gagnon et al., unpublished observations).

(3) A pAdCMV5-RFP/PS vector, which produces RFP under the control of the CMV promoter, was used to generate the control virus (AdCTRL) (D. Gagnon et al., unpublished observations).

For production of adenoviruses, $10^{6} 293 \mathrm{~A}$ cells cultured in a $60 \mathrm{~mm}$ dish were transfected with $3 \mu \mathrm{g}$ of linearized shuttle vector using lipofectin (Life Technologies, Carlsbad, CA). Immediately after transfection, Ad5- $\Delta$ PS virus at a multiplicity of infection (MOI) of $10^{6}$ pfu was added to each dish. Cells were lyzed $3 \mathrm{~d}$ later through cycles of freezing and thawing, and viral particles were separated from cell debris by centrifugation. Positive viral recombinants were identified by visualization of green fluorescent protein (GFP) or RFP production in 293A cells. Amplification of individual viral clones, their purification with the Adenopure system (Puresyn, Malvern, PA), as well as determination of viral titer by plaque assay were performed as described previously (Ogorelkova et al., 2004). The titers of the Ad-CTRL, Ad-Sim1, and Ad-shRNA-Sim 1 stocks were $2.1 \times 10^{12}, 1.8 \times 10^{12}$, and $2 \times 10^{12}$ $\mathrm{pfu} / \mathrm{ml}$, respectively.

Protein analysis. 293A cells were seeded at a density of $1 \times 10^{6}$ cells/60 $\mathrm{mm}$ dish and were infected at variable MOI. Cells were harvested $3 \mathrm{~d}$ after infection. For Western blot analysis, $20 \mu \mathrm{g}$ of total protein extract was loaded in each well of a 10\% SDS-polyacrylamide gel. Goat SIM1 polyclonal antibody (Santa Cruz Biotechnology, Santa Cruz, CA) and mouse actin monoclonal antibody (Abcam, Cambridge, MA) were diluted to 2 $\mathrm{mg} / \mathrm{ml}$ and $1: 10,000$, respectively. Secondary antibodies conjugated with peroxidase were visualized with the 3,3'-diaminobenzidine solution. Signal intensity of the bands was measured with the Fluorchem system (Alpha Innotech Corporation, San Leandro, CA).

Stereotaxic injections. Four or 5-week-old CD1 males, weighing between 25 and $30 \mathrm{~g}$, were housed individually in cages from at least $5 \mathrm{~d}$ before surgical interventions until they were killed. Each mouse given injections of the Ad-shRNA-Sim1 or the Ad-Sim1 virus was paired with a littermate that received injections on the same day of the Ad-CTRL virus. Mice used for the set of unilateral injections with the Ad-shRNA-Sim1 and Ad-CTRL viruses were maintained on a Harlan Teklad (Madison, WI) mouse breeder diet ( $10 \%$ crude fat), whereas mice used for the other injections were maintained on the Harlan Teklad global 19\% protein rodent diet ( $9 \%$ crude fat).

For injections, mice were anesthetized with $100 \mathrm{mg} / \mathrm{kg}$ ketamine (Ayerst, Guelph, Ontario, Canada) and $10 \mathrm{mg} / \mathrm{kg}$ xylazine (Ayerst) injected intraperitoneally. The skull was immobilized in a stereotaxic apparatus (David Kopf Instruments, Tujunga, CA). A $1.5 \mathrm{~mm}$ hole was created in the cranium by circular movements of hand-held Dumont 5-45 tweezers (Fine Science Tools, Vancouver, British Columbia, Canada). The stereotaxic coordinates for injection into the PVN were $0.85 \mathrm{~mm}$ caudal to bregma, $0.15 \mathrm{~mm}$ lateral to the midline, and $5.5 \mathrm{~mm}$ below the surface of the skull. Using a 31 gauge needle (Hamilton, Reno, NV) with a $5 \mathrm{ml}$ Hamilton microsyringe, $0.5 \mathrm{ml}$ of $4 \times 10^{7} \mathrm{pfu} / \mathrm{ml}$ or of $1.2 \times 10^{7} \mathrm{pfu} / \mathrm{ml}$ Ad5 vector preparations were injected into the PVN over a $10 \mathrm{~min}$ period. After vector delivery, the needle was left in place for $20 \mathrm{~min}$ to prevent reflux and slowly withdrawn in several steps, over $5 \mathrm{~min}$. Food intake and body weight were measured daily from the third or the fourth to the eighth day after the procedure.

Validation of injection site. Eight days after adenoviral injection, mice

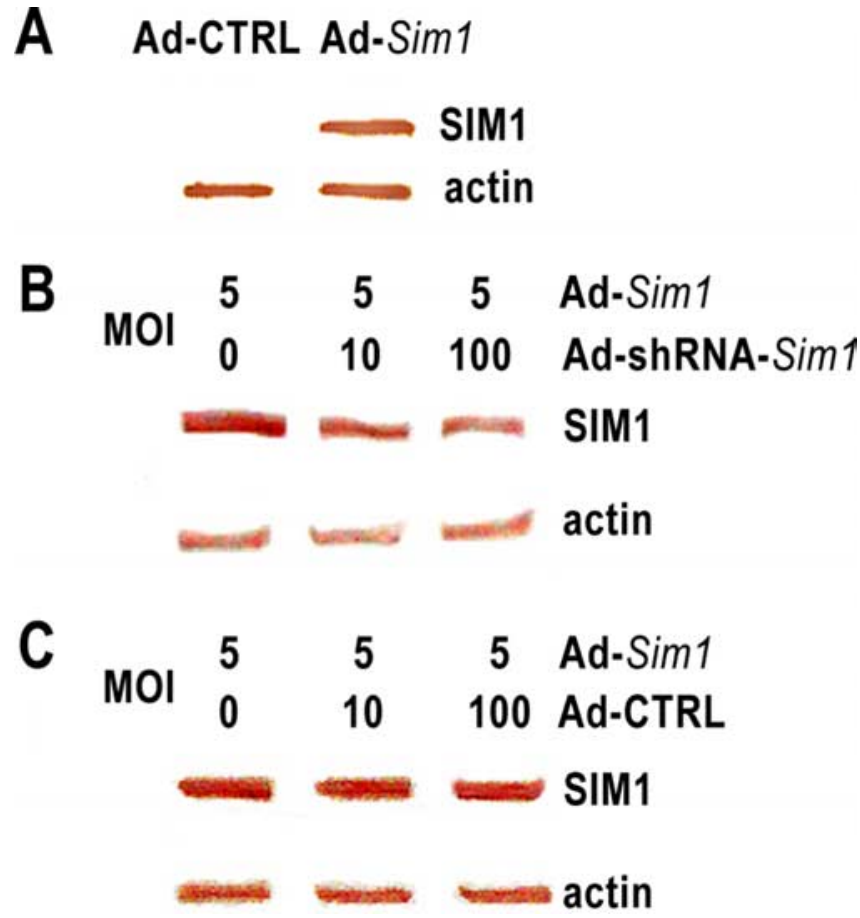

Figure 1. Western blot detection of SIM1 production in cultured 293A cells infected with adenoviruses. A, Production of SIM1 protein was detectable in cells infected with Ad-Sim 1 but not with Ad-CTRL. B, Cells were infected with Ad-Sim1 (at a constant MOI of 5) and/or AdshRNA-Sim 1 (at an MOl of 10 or 100). Coinfection with Ad-Sim1 and Ad-shRNA-Sim 1 resulted in a $40 \%$ decrease in SIM1 production when an $\mathrm{MOI}$ of 10 of the latter virus was used, whereas infection with Ad-shRNA-Sim 1 at an MOI of 100 decreased SIM1 production by $60 \%$. Infection by these viruses did not affect actin production. C, Cells were infected with Ad-Sim1 (at a constant MOI of 5) and/or Ad-CTRL (at an MOl of 10 or 100). Infection with Ad-CTRL did not affect the production of SIM1 protein by the Ad-Sim1 virus.

were anesthetized and perfused with paraformaldehyde $4 \%$ (Michaud et al., 2001). Brains were processed to produce $20 \mu \mathrm{M}$ coronal cryostat sections, as described previously (Michaud et al., 2001). Successful injection was defined by the presence of RFP- or GFP-producing cells in the PVN. In situ hybridization using a Sim 1 probe labeled by UTP-S ${ }^{35}$ incorporation was performed as described previously (Michaud et al., 1998). Sim1 expression in the injected and noninjected PVNs was compared by quantifying hybridization signals using the Image Pro software (Media Cybernetics, Silver Spring, MD) on every fourth section. For each mouse, the sum of arbitrary values for the total intensity of signals captured on one side of the PVN was calculated and compared with the sum of intensity values observed on the other side.

Statistical analysis. Results were expressed as means \pm SEM. Statistical significance was determined using a paired Student's $t$ test. We correlated changes in Sim1 expression and food intake by calculating the Pearson coefficient. For Sim1 expression, we used the average change of the four contiguous sections with the greatest differences between the injected and noninjected sides. For food intake, we used the average change of the 3 consecutive days with the greatest differences between mice that received the experimental viruses and mice that received the control virus.

\section{Results}

To modulate Sim1 expression levels in the PVN, we first generated three adenoviral vectors: Ad-shRNA-Sim1, which produces a shRNA directed against Sim1; Ad-Sim1, which expresses Sim1; and Ad-CTRL. We found that infection of 293A cells with the Ad-Sim 1 virus resulted in the abundant production of a SIM1 protein of the expected size (Fig. 1A). Coinfection of cells with Ad-Sim 1 and Ad-shRNA-Sim 1 resulted in a $60 \%$ decrease in the production of SIM1, whereas coinfection with Ad-Sim1 and Ad- 
CTRL did not affect the production of the SIM1 protein by the former virus (Fig. $1 B, C$ ). The CMV promoter used to direct Sim 1 expression is much stronger than the $\mathrm{H} 1$ promoter used to direct shRNA expression. This difference may explain why we needed 20 times more Ad-shRNA-Sim1 than Ad-Sim1 viral particles to achieve this $60 \%$ reduction.

We next used a protocol of stereotaxic injection to infect the PVN of mice with these adenoviruses. In a first series of experiments, we injected $20 \times 10^{6}$ Ad-shRNA-Sim 1 viral particles unilaterally into the PVN of 10 wild-type CD1 mice. Each of these mice was paired with a littermate that was injected on the same day with the same dose of the Ad-CTRL virus. After $4 \mathrm{~d}$ of recuperation from surgery, food intake and body weight were measured daily until the end of the eighth day when mice were killed. Two pairs of mice were excluded from the analysis because RFPproducing cells were not detected in the PVN of at least one of the littermates. There was no significant difference between the body weight of mice that received injections of the Ad-shRNA-Sim 1 or the Ad-CTRL virus before and after the injections (data not shown). Food intake was significantly increased from the fifth to the eighth day in mice given injections of the Ad-shRNA-Sim 1 virus compared with those given injections of the Ad-CTRL virus $(p<0.005 ; n=8$ mice for each virus) (Fig. $2 A)$. The peak of the adenoviral effect was reached on the sixth day when food intake was increased by an average of $22 \%$ in mice given injections of the Ad-shRNA-Sim 1 virus. The time course of the effect of AdshRNA-Sim 1 on food intake correlated with the fact that Ad5 transgene expression in mouse brain usually peaks from 4 to $7 \mathrm{~d}$ after infection and declines thereafter (Alisky and Davidson, 2004).

Sim1 expression levels in the infected and noninfected halves of the PVN were compared by in situ hybridization in these mice $8 \mathrm{~d}$ after the injection. Sim 1 expression was decreased by $29 \%$, on average, in the PVN halves infected with the Ad-shRNA-Sim1 virus ( $p<0.005 ; n=8$ mice for each side), whereas it was not significantly changed in PVNs infected with the control virus (Fig. 2B-E). Sim 1 expression in the PVN halves infected with Ad-shRNA-Sim 1 was decreased by $>20 \%$ in $63 \%$ of sections, indicating that only a subset of the PVN was infected. Histological examination did not reveal grossly abnormal cell density within the infected PVN (Fig. 2B). We observed a correlation between changes in Sim1 expression levels induced by the AdshRNA-Sim 1 virus and food intake $(p<0.05)$. Of note, injection of $6.0 \times 10^{6}$ Ad-shRNA-Sim 1 particles did not affect food intake compared with the injection of the same dose of the Ad-CTRL virus (data not shown; $n=4$ mice for each virus). The initial dose of $20 \times 10^{6}$ viral particles was thus used for the remaining experiments.

Using the same protocol of stereotaxic injection, we next compared the effect of the Ad-Sim1 and Ad-CTRL viruses on food intake. Nine pairs of mice were given injections, but two were excluded from the analysis because GFP- or RFP-producing cells were not detectable in the PVN of at least one littermate. There was no significant difference between the body weight of mice given injections of the Ad-Sim1 or the Ad-CTRL before and after the injections (data not shown). Food intake was decreased from the sixth to the eighth day after the injection in mice infected with the Ad-Sim1 virus compared with those infected with the AdCTRL virus ( $p<0.005 ; n=7$ mice for each virus) (Fig. $3 A$ ). The peak of the adenoviral effect was reached on the seventh day when food intake was decreased by $20 \%$, on average, in mice given injections of the Ad-Sim 1 virus. Of note, food intake of mice infected with Ad-CTRL increased slightly but not significantly
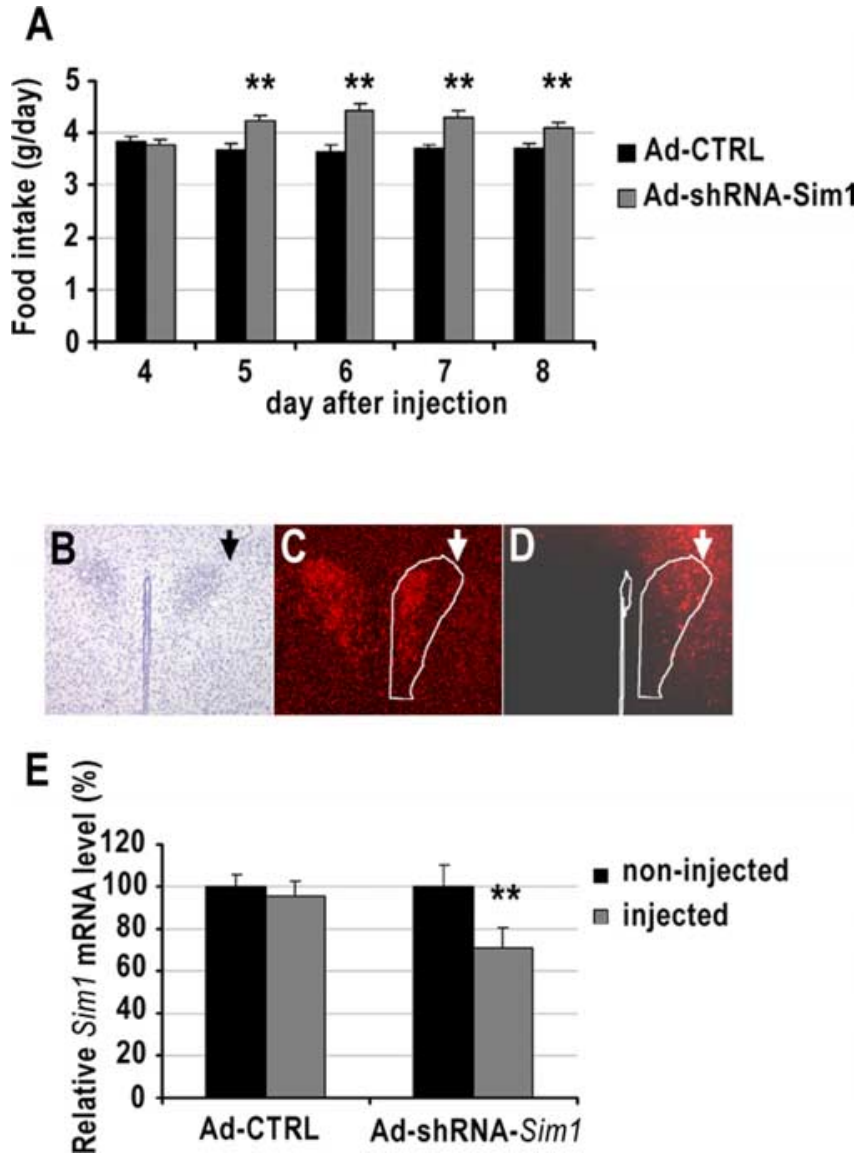

Figure 2. Unilateral infection of the PVN with the Ad-shRNA-Sim1 virus increases food intake. $\boldsymbol{A}$, Daily food intake of mice infected with Ad-CTRL or Ad-shRNA-Sim $1\left({ }^{* *} p<0.005 ; n=\right.$ 8 mice for each virus). $\boldsymbol{B}-\boldsymbol{D}$, Representative coronal sections through the PVN of a mouse infected with Ad-shRNA-Sim1. The virus was injected into the right side of the PVN, and the mouse was killed $8 \mathrm{~d}$ after the infection. $\boldsymbol{B}$ and $\boldsymbol{C}$ are bright- and dark-field views, respectively, of the same section, which was hybridized with a $\operatorname{Sim} 1$ probe, whereas $\boldsymbol{D}$ is adjacent to $\boldsymbol{B}$ and $\boldsymbol{C}$ and shows RFP production. The arrow in $\boldsymbol{B}-\boldsymbol{D}$ is positioned on the tract of the needle with its pointed extremity corresponding to the deepest end of the tract. $\boldsymbol{E}$, Quantification of Sim 1 expression by in situ hybridization. The Ad-CTRL or Ad-shRNA-Sim 1 virus was always injected on the right side. The sum of intensity values for each side of the PVN was compared. The values corresponding to the injected side are expressed relatively to those corresponding to the noninjected side, which are set at $100 \%\left({ }^{* *} p<0.005 ; n=8\right.$ mice for each side $)$. Error bars indicate SEM.

after the injection. We observed this effect in another set of experiments (see below) (Fig. 4) but not in the first one (Fig. 2). In situ hybridization studies indicated that Sim 1 expression was increased, on average, by $19 \%$ in the infected half of the PVN of mice that received the Ad-Sim 1 virus, but it was not changed in those that received the control virus (Fig. $3 B-E$ ). Sim1 expression in the PVN halves infected with Ad-Sim 1 was increased by $>20 \%$ in $57 \%$ of sections, suggesting again that only a subset of the PVN was infected. We observed a correlation between changes in Sim 1 expression levels induced by the Ad-Sim 1 virus and food intake $(p<0.05)$.

To explore the impact of more extensive changes in Sim 1 expression levels on food intake, we performed a last set of experiments in which both sides of the PVN were injected with the Ad-shRNA-Sim1, Ad-Sim1, or Ad-CTRL virus on the same day in trios of littermates. RFP- or GFP-producing cells were detectable in both sides of the PVN in all of the injected mice. Bilateral injection of the Ad-shRNA-Sim 1 virus into the PVN significantly increased food intake from the fifth to the eighth day after the 

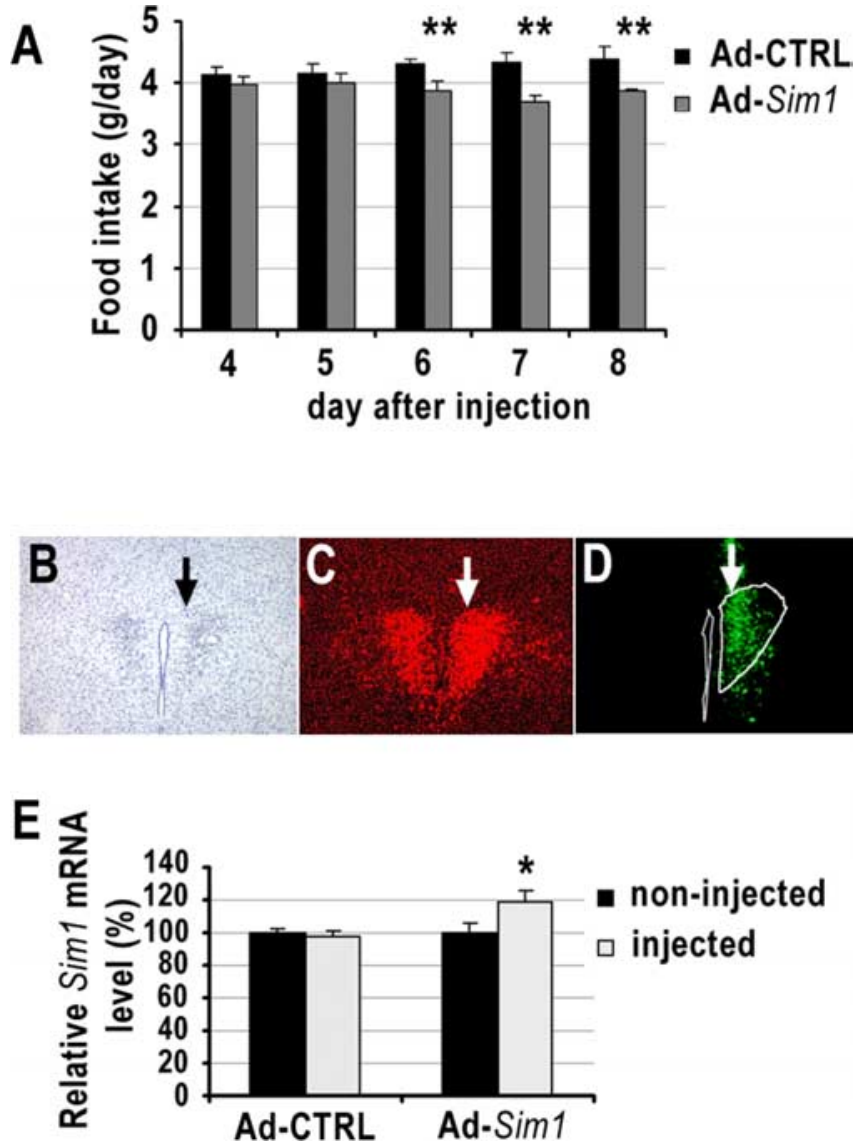

Figure 3. Unilateral infection of the PVN with the Ad-Sim 1 virus decreases food intake. $A$, Daily food intake of mice infected with the Ad-CRTL or the Ad-Sim 7 virus $\left({ }^{* *} p<0.005 ; n=7\right.$ mice for each virus). $\boldsymbol{B}-\boldsymbol{D}$, Representative coronal sections through the PVN of a mouse that was infected with the Ad-Sim1. The virus was injected into the right side of the PVN, and the mouse was killed $8 \mathrm{~d}$ after the infection. $\boldsymbol{B}$ and $\boldsymbol{C}$ are bright- and dark-field views, respectively, of the same section, which was hybridized with a $\operatorname{Sim} 1$ probe, whereas $\boldsymbol{D}$ is adjacent to $\boldsymbol{B}$ and $\boldsymbol{C}$ and shows GFP production. The arrow in $\boldsymbol{C}$ and $\boldsymbol{D}$ is positioned on the tract of the needle with its pointed extremity corresponding to the deepest end of the tract. $\boldsymbol{E}$, Quantification of $\operatorname{Sim} 1$ expression by in situ hybridization. The Ad-CTRL or the Ad-Sim 1 virus was always injected on the right side. The sum of intensity values for each side of the PVN was compared. The values corresponding to the injected side are expressed relatively to those corresponding to the noninjected side, which are set at $100 \%\left({ }^{*} p<0.005 ; n=8\right.$ mice for each side). Error bars indicate SEM.

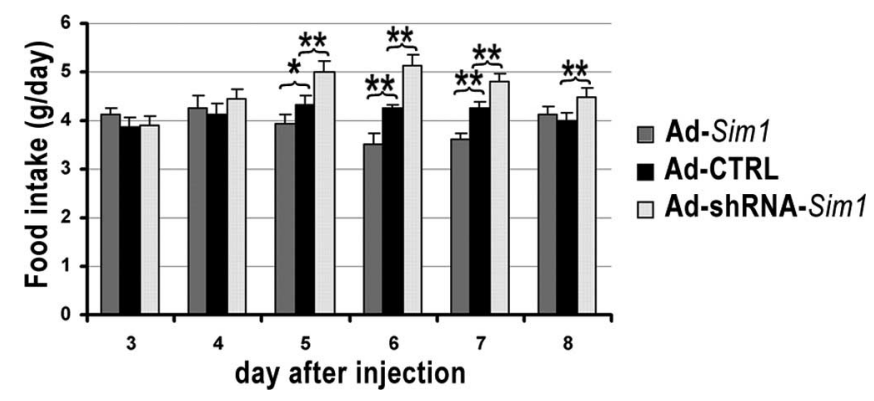

Figure 4. Bilateral infection of the PVN with adenoviruses that modulate $\operatorname{Sim} 1$ expression levels affects food intake. Daily food intake of mice infected with the Ad-Sim1, Ad-shRNA-Sim1, or the Ad-CTRL virus ( ${ }^{*} p<0.05 ;{ }^{* *} p<0.005 ; n=7$ mice for each virus). Error bars indicate SEM.

procedure ( $p<0.005 ; n=7$ mice for each virus), whereas injection of the Ad-Sim1 virus significantly decreased food intake from the fifth to the eighth day $(p<0.05 ; n=7$ mice for each virus) (Fig. 4). The most dramatic changes were observed on the sixth day with the Ad-shRNA-Sim 1 virus increasing food intake by an average of $21 \%$ and the Ad-Sim 1 virus decreasing food intake by an average of $17 \%$, compared with the effect of the Ad-CTRL virus. Although bilateral infection of the PVN by these viruses did not have a more pronounced effect than unilateral infection, this third set of injections further suggests that modulation of Sim 1 expression levels specifically affects food intake.

\section{Discussion}

We have reported previously that $\operatorname{Sim} 1$ is required for the development of the PVN (Michaud et al., 1998). The experiments reported here indicate that $\operatorname{Sim} 1$ plays a second role in the PVN, acting along a physiological pathway to control food intake. We cannot, however, exclude the possibility that the structural defects identified in the PVN of Sim $1^{+/-}$mice may also contribute to their hyperphagia. For instance, the obesity of other models, such as leptin-deficient mice, may be explained by a combination of developmental and physiological defects (Bouret et al., 2004). The use of a conditional mutant allele of $\operatorname{Sim} 1$ would be necessary to dissect the respective contributions of such factors to the obesity caused by $\operatorname{Sim} 1$ haploinsufficiency.

The Sim1 gene copy number has a major influence on food intake in mice and in humans (Holder et al., 2000, 2004; Michaud et al., 2001). Our paradigm of stereotaxic injection of adenoviruses into the PVN does not easily permit correlations between physiological end points and levels of gene expression, because only a subset of the PVN is infected. However, it is noteworthy that reduction in Sim1 expression in only a limited number of PVN cells is sufficient to affect food intake. Of note, the impact of bilateral infection of the PVN was not greater than that of unilateral infection. Several explanations could be proposed to account for this observation. For instance, a component of the Sim 1 pathway could be found in limiting amounts in PVN cells, restricting the impact of an increase in Sim1 expression levels. Also, the impact on food intake of small changes in the activity of the Sim 1 pathway could reach a plateau either because of the intrinsic properties of the pathway or because of the existence of counterregulatory loops.

Our observation that Sim1 functions in the PVN to influence food intake should be interpreted in the context of a great body of work establishing this nucleus as a critical regulator of energy balance (Choi and Dallman, 1999; Cowley et al., 1999). The PVN appears to integrate several hypothalamic and extrahypothalamic signals to provide a major hypothalamic output for the regulation of energy intake through its projections to the brainstem (Blevins et al., 2004; Rinaman et al., 2005). Sim1 is also expressed in cells scattered in the anteroventral hypothalamus as well as in the nucleus of the lateral olfactory tract (Balthasar et al., 2005). These cells have not been formally associated with the regulation of food intake. Although we cannot exclude the possibility that $\operatorname{Sim} 1$ also functions in these cells, it seems likely that the PVN is its main site of action for the control of food intake.

The control of food intake and energy expenditure by the PVN seems to involve divergent pathways. For instance, the NPY pathway harbored by the PVN influences feeding, at least in part, through projections to the rostral nucleus of the solitary tract, located in the brainstem, whereas its effects on thermogenesis in the brown adipose tissue has been suggested to involve another neural site (Kotz et al., 1998). Moreover, a recent study using a conditional allele of $M c 4 r$, which codes for a melanocortin receptor, strongly suggests the existence of divergent melanocortin pathways: the melanocortin pathway in the PVN controls food intake, whereas a melanocortin pathway located elsewhere con- 
trols energy expenditure (Balthasar et al., 2005). Although energy expenditures were not measured in this study, our previous work indicates that $\operatorname{Sim} 1$ haploinsufficiency affects food intake without a major effect on energy expenditure (Michaud et al., 2001). Together, our results would be consistent with the existence of a specific pathway in the PVN that regulates food intake independently of energy expenditure.

Mice with a decrease in Sim1 or of Mc4r show phenotypic similarities, both with early-onset hyperphagia, increased linear growth, and enhanced sensitivity to diet-induced obesity (Michaud et al., 2001; Holder et al., 2004). One possibility would be that $\operatorname{Sim} 1$ regulates a component of the melanocortin pathway located in the PVN. It has been suggested previously that Sim1 haploinsufficiency does not affect $M c 4 r$ expression in the hypothalamus (Holder et al., 2004). Therefore, if Sim1 regulates the $M c 4 r$ pathway, it would do so by acting downstream of $M c 4 r$. An interaction between Sim1 and the Mc4r pathway could thus be explored by overexpressing Sim1 in mice with a decrease in melanocortin signaling or by activating pharmacologically this pathway in mice with a decrease in Sim 1 gene dosage.

SIM1 can be added to a growing list of PAS domain proteins that are involved in the regulation of physiological processes acting at the interface between the environment and the internal milieu ( $\mathrm{Gu}$ et al., 2000). The activity of some PAS proteins has been shown to be regulated by small ligands. It is tempting to speculate that $\operatorname{Sim} 1$ is regulated by a similar mechanism, representing an interesting target for pharmacological manipulation.

\section{References}

Alisky JM, Davidson BL (2004) Gene transfer to brain and spinal cord using recombinant adenoviral vectors. Methods Mol Biol 246:91-120.

Balthasar N, Dalgaard LT, Lee CE, Yu J, Funahashi H, Williams T, Ferreira M, Tang V, McGovern RA, Kenny CD, Christiansen LM, Edelstein E, Choi B, Boss O, Aschkenasi C, Zhang CY, Mountjoy K, Kishi T, Elmquist JK, Lowell BB (2005) Divergence of melanocortin pathways in the control of food intake and energy expenditure. Cell 123:493-505.

Blevins JE, Schwartz MW, Baskin DG (2004) Evidence that paraventricular nucleus oxytocin neurons link hypothalamic leptin action to caudal brain stem nuclei controlling meal size. Am J Physiol Regul Integr Comp Physiol 287:R87-R96.

Bouret SG, Draper SJ, Simerly RB (2004) Trophic action of leptin on hypothalamic neurons that regulate feeding. Science 304:108-110.

Choi S, Dallman MF (1999) Hypothalamic obesity: multiple routes mediated by loss of function in medial cell groups. Endocrinology 140:4081-4088.

Cowley MA, Pronchuk N, Fan W, Dinulescu DM, Colmers WF, Cone RD (1999) Integration of NPY, AGRP, and melanocortin signals in the hypothalamic paraventricular nucleus: evidence of a cellular basis for the adipostat. Neuron 24:155-163.

Elahi SM, Oualikene W, Naghdi L, O'Connor-McCourt M, Massie B (2002) Adenovirus-based libraries: efficient generation of recombinant adenoviruses by positive selection with the adenovirus protease. Gene Ther 9:1238-1246.

Gu YZ, Hogenesch JB, Bradfield CA (2000) The PAS superfamily: sensors of environmental and developmental signals. Annu Rev Pharmacol Toxicol 40:519-561.

Holder Jr JL, Butte NF, Zinn AR (2000) Profound obesity associated with a balanced translocation that disrupts the SIM1 gene. Hum Mol Genet 9:101-108.

Holder Jr JL, Zhang L, Kublaoui BM, DiLeone RJ, Oz OK, Bair CH, Lee YH, Zinn AR (2004) Siml gene dosage modulates the homeostatic feeding response to increased dietary fat in mice. Am J Physiol Endocrinol Metab 287:E105-E113.

Kotz CM, Briggs JE, Grace MK, Levine AS, Billington CJ (1998) Divergence of the feeding and thermogenic pathways influenced by NPY in the hypothalamic PVN of the rat. Am J Physiol 275:R471-R477.

Michaud JL, Rosenquist T, May NR, Fan C-M (1998) Development of neuroendocrine lineages requires the bHLH-PAS transcription factor SIM1. Genes Dev 12:3264-3275.

Michaud JL, Boucher F, Melnyk A, Gauthier F, Goshu E, Levy E, Mitchell GA, Himms-Hagen J, Fan C-M (2001) Siml haploinsufficiency causes hyperphagia, obesity and reduction of the paraventricular nucleus of the hypothalamus. Hum Mol Genet 10:1465-1473.

Ogorelkova M, Elahi SM, Gagnon D, Massie B (2004) DNA delivery to cells in culture: generation of adenoviral libraries for high-throughput functional screening. Methods Mol Biol 246:15-27.

Rinaman L, Vollmer RR, Karam J, Phillips D, Li X, Amico JA (2005) Dehydration anorexia is attenuated in oxytocin-deficient mice. Am J Physiol Regul Integr Comp Physiol 288:R1791-R1799.

Swanson LW, Sawchenko PE (1983) Hypothalamic integration: organization of the paraventricular and supraoptic nuclei. Annu Rev Neurosci 6:269-324 